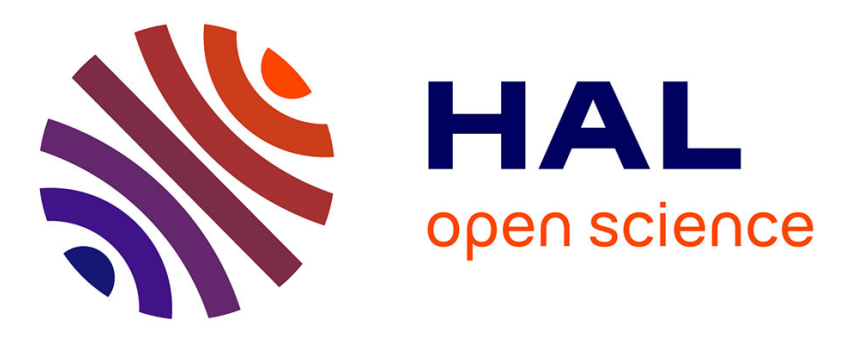

\title{
An expanding cavity model incorporating pile-up and sink-in effects
}

\author{
Xavier Hernot, Olivier Bartier
}

\section{To cite this version:}

Xavier Hernot, Olivier Bartier. An expanding cavity model incorporating pile-up and sink-in effects. Journal of Materials Research, 2011, 27 (1), pp.132-140. 10.1557/jmr.2011.394 . hal-00861060

\section{HAL Id: hal-00861060}

\section{https://hal-univ-rennes1.archives-ouvertes.fr/hal-00861060}

Submitted on 11 Sep 2013

HAL is a multi-disciplinary open access archive for the deposit and dissemination of scientific research documents, whether they are published or not. The documents may come from teaching and research institutions in France or abroad, or from public or private research centers.
L'archive ouverte pluridisciplinaire HAL, est destinée au dépôt et à la diffusion de documents scientifiques de niveau recherche, publiés ou non, émanant des établissements d'enseignement et de recherche français ou étrangers, des laboratoires publics ou privés. 


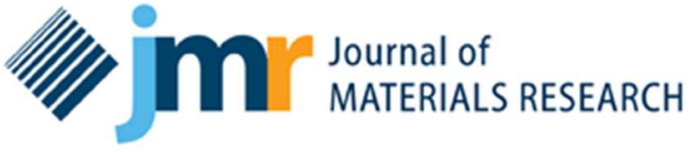

\section{An expanding cavity model incorporating pile-up and sink-in effects}

\begin{tabular}{|r|l|}
\hline Journal: & Journal of Materials Research \\
\hline Manuscript ID: & JMR-2011-0279.R2 \\
\hline Manuscript Type: & Focus Issue: Instrumented Indentation \\
\hline Date Submitted by the Author: & 14-Oct-2011 \\
\hline Complete List of Authors: & $\begin{array}{l}\text { hernot, xavier; université de Rennes 1, LARMAUR-Indentation } \\
\text { bartier, olivier; Université de Rennes1, LARMAUR-Indentation E.A. 4282 }\end{array}$ \\
\hline Key Words: & nano-indentation, simulation, hardness \\
\hline & \\
\hline
\end{tabular}

SCHOLARONE ${ }^{\text {IM }}$

Manuscripts 


\title{
An expanding cavity model incorporating pile-up and sink-in effects
}

\author{
X. Hernot ${ }^{a}$,O. Bartier ${ }^{b}$ \\ ${ }^{a}$ L.A.R.M.A.U.R-Indentation, E.A. 42.82, Bât 10B, Université de Rennes 1, Campus de Beaulieu, 35402 \\ Rennes Cedex, France. Olivier.bartier@univ-rennes1.fr \\ ${ }^{\mathrm{b}}$ L.A.R.M.A.U.R-Indentation, E.A. 42.82, Bât 10B, Université de Rennes 1, Campus de Beaulieu, 35402 \\ Rennes Cedex, France. Xavier.hernot@univ-rennes1.fr \\ Corresponding author: Xavier.Hernot, Tel: +33 22323 6148; fax: +33 223236111 E-mail address: \\ Xavier.Hernot@univ-rennes1.fr L.A.R.M.A.U.R, E.A. 410, Bât 10B, Université de Rennes 1, Campus de \\ Beaulieu, 35402 Rennes Cedex, France.
}

\begin{abstract}
.
A new expanding cavity model (ECM) for describing conical indentation of elastic ideally-plastic material is developed. For the proposed ECM, it is assumed that the volume of material displaced by the indenter is equal to the volume loss, due to elastic deformation, in the material and depends on the pile-up or sink-in. It was shown that the proposed ECM matches very well numerical data in the final portion of the transition regime for which the contact pressure lies between approximately $2.5 \mathrm{Y}$ and $3 Y$. For material of large $E / Y$ ratio, the new ECM also matches very well numerical data in the plastic-similarity regime. For material of smaller $E / Y$ ratio, the proposed ECM gives better results than the Johnson's ECM because pileup or sink-in is taken into account.
\end{abstract}

\section{INTRODUCTION}

Elastic, elastic-plastic and fully plastic regimes were observed for conical indentation. ${ }^{1-4}$ The deformation process produced during conical indentation is well described by Love ${ }^{1}$ when the regime is elastic. When plasticity occurs, it is more complex to describe indentation responses because the material of the sample exhibits multiaxial stress conditions with high gradients and large elastic-plastic strains in the indentation region. Strong non-linearities are induced by the unilateral contact and the involved large 
displacements. A Pile-up can be also developed during the indentation test. Because of the complex nature of the strain field produced by an indentation, the first analytical solutions have been limited to rigidideally plastic or elastic materials. For the former, Tabor $^{3}$ has shown that the mean indentation pressure $H$ is related to the material flow stress $Y$ by

$$
H=C_{f} Y
$$

where $C_{f}$ is a constant, called "constraint factor", depending upon the geometry of the indenter with a value near three.

Slip-line pattern also leads to Eq. (1) for frictionless cone penetrating a flat surface if the semi apex angle of the cone is large $\mathrm{e}^{2}$. It was shown that this simple hardness theory is deficient for highly elastic materials. ${ }^{5}$ Attempts were made to correlate indentation pressure in elastic-plastic materials using the theoretical analysis of the expansion of a cylindrical or spherical cavity by an internal pressure. ${ }^{5-7}$ The most famous of these was by Johnson. ${ }^{8}$ He considered the indenter to be encased in a hemispherical core of radius $a$ which replaces the cavity in Hill's model. ${ }^{9}$ A hydrostatic uniform pressure is assumed to exist throughout the core, equal in magnitude to the mean indentation pressure. In the Johnson's model ${ }^{8}, \mathrm{H}$ is related not only to the yield stress (Y) but also to the Young's modulus of the indented material (E) and the geometry of the indenter as well. The expanding cavity model involves certain key assumptions such as a not purely hydrostatic stress in the material immediately below the indenter. This assumption gives better agreement with experimental data but introduces other inconsistencies. The basic difficulty is that the assumption of a true hydrostatic pressure in the core is not valid. Other shortcomings of this model have been pointed out in the literature and some remedial measures have been suggested. ${ }^{10-12} \operatorname{Studman}^{10}$ pointed out a step-discontinuity in the stress field at the core-plastic zone interface. An improved result is obtained if it is assumed that the hemispherical core is a region in which the stresses are changing from purely hydrostatic to values satisfying the Von Mises yield criterion. ${ }^{10}$ This modification introduces also other inconsistencies. Another defect pertains to the non-vanishing normal traction outside the contact 
zone on the specimen surface, which is an outcome of applying Hill's solution ${ }^{9}$ for a spherical cavity directly to the indentation problem. There have been two elegant treatments to account for the specimen surface. The first is Chiang's revised expanding model. ${ }^{11}$ They calculated an auxiliary field generated by the exact negative tractions applied on the surface of a half space, and then they could represent the free surface by summing the auxiliary field with the original solution. The second model to account for the surface effect is based on the Yoffe' surface inclusion model. ${ }^{13}$ According to this model, Feng et al ${ }^{12}$ proposed that the stress distribution outside the contact-induced plastic zone can be estimated by the superposition of a Hertzian field and an embedded center of dilatation (ECD) in a half-space, while the residual stress distribution can be estimated by the ECD field alone. Because of their complexity, these approaches have not been widely used in practice.

Notwithstanding these inherent assumptions and limitations, the expanding cavity model (ECM) is still being employed to interpret indentation data in ductile solids. ${ }^{14,15}$ The reason for the enduring popularity of this model is its simplicity and ability to predict with reasonable accuracy important experimental results such as the indentation stress versus strain curve, evolution of plastic zone size with load and stress distribution beneath the indenter. ${ }^{2,8,14,15}$

The objective of this paper is to provide a new expanding cavity model (ECM) for describing conical indentation. We show that the Johnson's expanding cavity model ${ }^{8}$ based on the conservation of volume during an increment of penetration can be replaced by an ECM based on the equality between the displaced volumes and the volume loss in the indented material. In a first step, it is assumed that the volume of material displaced by the indenter is equal to the volume loss, due to elastic deformation, in the core, plastic zone and elastic zone. In a second step, we propose a new formulation of ECM for materials that exhibit pile-up or sink-in. It was shown that the Johnson's ECM fits very well experiment data in the elastic-plastic regime, i.e. the transitional regime where $\mathrm{H}$ lies between $0.5 \sim 3 \mathrm{Y} .{ }^{2,10,14}$ The purpose of this paper is also to show that the results of ECM can be in agreement with numerical results in regimes, whose contact response is more plastically dominated, if pile-up or sink-in is taken into account in the model. 


\section{JOHNSON'S EXPANDING CAVITY MODEL (ECM)}

It is assumed that the elastic-plastic regime begins when the yield point is exceeded and occurs up to the fully plastic regime for which the elasticity is considered as negligible. In this regime, the plastic zone is contained by material which remains elastic. For the elastic-plastic regime, $\mathrm{Marsh}^{4}$ and Johnson ${ }^{8}$ suggested that the material in the indented zone can be viewed as being encased in an expanding hemispherical core. The proposed model is based on the compatibility between the volumetric expansion of the core and the volume of material displaced by the indenter. In this context, it was considered that (1) the hemispherical core is incompressible and subjected to an internal pressure, (2) the radius of the core, $a$ , is equal to the contact radius and (3) the hydrostatic stress within the core is equal to the radial stress at the core boundary (Fig. 1).

FIG. 1: Schematic illustrating the Johnson's ECM for conical indentation.

Outside the core it is assumed that the stresses and displacements have radial symmetry. The stresses in the plastic zone, $a \leq r \leq c$, are given by Hill' ${ }^{9}$ :

$$
\frac{\sigma_{r}}{Y}=-2 \ln \left(\frac{c}{r}\right)-\frac{2}{3} \quad \frac{\sigma_{\theta}}{Y}=-2 \ln \left(\frac{c}{r}\right)+\frac{1}{3}
$$

In the elastic zone, $r \geq c$ :

$$
\frac{\sigma_{r}}{Y}=-\frac{2}{3}\left(\frac{c}{r}\right)^{3} \quad \frac{\sigma_{\theta}}{Y}=\frac{1}{3}\left(\frac{c}{r}\right)^{3}
$$

At the interface between core and the plastic zone, the hydrostatic stress in the core is just equal to the radial component of stress in the external zone, $\sigma_{r}$ : 


$$
\frac{\bar{P}}{Y}=-\frac{\sigma_{r}}{Y}=2 \ln \left(\frac{c}{a}\right)+\frac{2}{3}
$$

Based on the radial displacements within the plastic zone given by Hill ${ }^{9}$, conservation of volume and concept of geometrical similarity of the indentation, the ratio of the plastic zone size to the contact radius is:

$$
\left(\frac{c}{a}\right)^{3}=\frac{1}{6(1-v)}\left[\frac{E \tan \beta}{Y}+4(1-2 v)\right]
$$

where $\beta$ is the inclination of the face of the cone to the surface.

\section{NEW FORMULATION OF THE EXPANDING CAVITY MODEL}

\section{A) Ratio of the plastic zone size to the contact radius}

For the new formulation, it is assumed that the volume of material displaced by the indenter is equal to the volume loss, due to elastic deformation, in the core, plastic zone and elastic zone:

$$
\Delta V_{i}=\Delta V_{c}+\Delta V_{p}+\Delta V_{e}
$$

Where $\Delta V_{i}$ is the volume of material displaced by the indenter, $\Delta V_{c}, \Delta V_{p}$ and $\Delta V_{e}$ are the volume loss due to elastic deformation, respectively in the core, plastic zone and elastic zone.

For conical indenters, the volume of material displaced by the indenter is:

$$
\Delta V_{i}=\frac{\pi}{3} a^{3} \tan \beta
$$

The volume loss in the elastic zone is:

$$
\Delta V_{e}=2 \pi c^{2} u_{r=c}
$$

where $u_{r=c}$ is the radial displacement at the elastic-plastic interface.

The volume loss in the plastic zone is:

$$
\Delta V_{p}=2 \pi a^{2} u_{r=a}-\Delta V_{e}
$$

where $u_{r=a}$ is the radial displacement at the core-plastic zone interface. 
Using the Hill's solution for $u_{r=a}$ in Eq. (9) leads to:

$$
\Delta V_{e}+\Delta V_{p}=2 \pi a^{2} \frac{Y}{E} a\left[(1-v)\left(\frac{c}{a}\right)^{3}-\frac{2}{3}(1-2 v)\left(1+3 \ln \frac{c}{a}\right)\right]
$$

For the proposed formulation of ECM, constant hydrostatic pressure in the core and continuous stress field at the interface between core and plastic zone are assumed, i.e:

$$
\sigma_{r}\left(r=a^{-}\right)=\sigma_{r}\left(r=a^{+}\right) ; \quad \sigma_{\theta}\left(r=a^{-}\right)=\sigma_{\theta}\left(r=a^{+}\right)
$$

With these assumptions, the constant hydrostatic pressure in the core is equal to:

$$
\bar{p}=2 Y \ln \frac{c}{a}
$$

On contrary to the Johnson's formulation, the stress in the core is not purely hydrostatic in our formulation. With the concept of geometric similarity of the indentation, i.e: $c / a=$ constant, $\bar{p}$ is constant during indentation.

The advantage of the proposed formulation is that continuity in the Von Mises stress field exists at the interface between core and plastic zone on contrary to the classical formulation of the expanding cavity model $^{8}$. The assumption of continuous stress field at the interface between core and plastic zone is incompatible with the assumption of hydrostatic core. In consequence, the mean indentation pressure can not be determined starting from the hydrostatic pressure.

From Eq. (12) it follows that the volume loss in the core is:

$$
\Delta V_{c}=2 \pi a^{3}(2-\tan \beta) \frac{(1-2 v)}{E} Y \ln \frac{c}{a}
$$


Substituting Eqs. (7), (10) and (13) into Eq. (6) then leads to

$$
\left(\frac{c}{a}\right)^{3}=\frac{1}{6} \tan \beta \frac{E}{Y(1-v)}+\frac{1-2 v}{1-v}\left(\frac{2}{3}-\tan \beta \ln \frac{c}{a}\right)
$$

Notice that, for low cone angle, Eq. (14) is similar to the Johnson's formulation (Eq. 5).

Hernot and Pichot ${ }^{16}$ show that the degree of deformation at the transition between the elastic-plastic regime and plastic regime depends on the normalized deformation $\Lambda^{\prime}$. In the case of conical indentation $\Lambda^{\prime}$ is:

$$
\Lambda^{\prime}=\frac{1}{2(1-v)} \frac{E}{Y} \tan \beta
$$

It thus follows that:

$$
\left(\frac{c}{a}\right)^{3}=\frac{\Lambda^{\prime}}{3}+\frac{2}{3} \frac{1-2 v}{1-v}
$$

\section{B) Indentation Pressure}

The equilibrium of the core leads to the indentation load:

$$
F=\pi a^{2} H=\pi a^{2} Y\left(\frac{2}{3}+2 \ln \frac{c}{a}\right)
$$

where $H$ is the indentation pressure.

The Johnson's formulation ${ }^{8}$, with a condition of purely hydrostatic stress in all parts of the core, is similar to Eq. (17). It was shown that this equation is inadequate to fit indentation experiments. ${ }^{2,}{ }^{10}$ Indeed, in Johnson's paper his theoretical curve lies completely beneath the data points by a roughly constant amount up to pressures where fully plastic behaviour ensues. For Johnson ${ }^{2}$, the problem is due to the fact that the stress in the material immediately below the indenter is not purely hydrostatic and thus the indentation pressure is not equal to the purely hydrostatic stress. Johnson modified his formulation in order to obtain a 
best estimate of the indentation pressure. In a similar way, Eq. (17) is modified in order to obtain better agreement between theoretical and experimental results:

$$
F=\pi a^{2} H=\pi a^{2} Y \frac{2}{3}\left(2+3 \ln \frac{c}{a}\right)
$$

\begin{abstract}
Because of the proposed assumptions for the new ECM, it is not possible to keep the same arguments that the Johnson arguments in order to explain the modification added to Eq. (17).

For the authors, Eq. (17) gives bad agreement with the experimental data because the proposed assumptions only give an approximation to the real system. Eqs. (2) and (3) are obtained with the assumption that the indentation of an elastoplastic half-space is equivalent to a spherical cavity expanding in an infinite medium. However, the spherical symmetry around a cavity in an infinite medium does not match the symmetry around an indentation on a half-space involving a free surface. For example, $\sigma_{\theta}$ must be equal to zero on the free surface and it is not the case by using the Eq. (2).
\end{abstract}

\title{
IV. NEW FORMULATION OF ECM CONSIDERING PILE-UP AND SINK-IN
}

Johnson performed his study with the assumption that no piling-up or no sinking-in occurs during indentation. We propose, in this section, a new formulation of ECM for materials that exhibit pile-up or sink-in.

For materials that exhibit pile-up, it is assumed that the volume of material displaced by the indenter is equal to the pile-up volume and the volume loss, due to elastic deformation, in the core, plastic zone and elastic zone (Fig. 2(a)).

For materials that exhibit sink-in, it is assumed that the volume of material displaced by the indenter and the sink-in volume are equal to the volume loss, due to elastic deformation, in the core, plastic zone and elastic zone (Fig. 2(b)). 
a) case of piling up

b) case of sinking-in

FIG. 2: Schematic illustrating elastic-plastic indentation as idealized by the expanding cavity model considering surface deformation. (a) pile-up and (b) sink-in.

\section{A) Materials that exhibit pile-up}

The equality between the displaced volumes and the volume loss in the indented material is:

$$
\Delta V_{i}-\Delta V_{p u}=\Delta V_{c}+\Delta V_{p}+\Delta V_{e}
$$

Where $\Delta V_{p u}$ is the volume of the pile-up.

The radius of the core is equal to $a_{c}$ and the volume of material displaced by the indenter is calculated starting from $a$ (Eq. 7). The volume in the pile-up can thus be determined by geometrical conditions. The volume of the pile-up is calculated from:

$$
C^{1}=\frac{h_{c}}{h}
$$

Where $h$ is the penetration depth and $h_{c}$ is the contact height.

In this equation, $C^{l}$ quantifies the degree of pile-up and sink-in during the indentation test; $C^{l}>1$ indicates pile-up, whereas, $C^{l}<1$ accounts for sink-in.

The volume in the pile-up is divided into two parts. The first part corresponds to the volume of pile-up calculated for $r<a_{c}$ and the second part corresponds to the volume calculated for $r>a_{c}$. The pile-up volume 
is obtained by rotating the surface shown Fig. 2 around symmetry $z$-axis. For the second part of the pile-up volume, only a fraction, $\alpha$, of the right angled triangle of legs $\left(c-a_{c}\right)$ and $\left(h_{c}-h\right)$ is considered. The value of $\alpha$ will be defined thereafter.

Following the present geometrical considerations, the volume of the pile-up is:

$$
\begin{aligned}
& \Delta V_{p u}=-\frac{\pi}{3} a_{c}^{3} \tan \beta\left(1-\frac{1}{C^{1}}\right)\left(-2+\frac{1}{C^{1}}+\left(\frac{1}{C^{1}}\right)^{2}\right) \\
& +\alpha \frac{\pi}{3} a_{c}^{3} \tan \beta\left(1-\frac{1}{C^{1}}\right)\left[-2+\frac{c}{a_{c}}+\left(\frac{c}{a_{c}}\right)^{2}\right]
\end{aligned}
$$

It thus follows that:

$$
\Delta V_{i}-\Delta V_{p u}=\frac{\pi}{3} a_{c}^{3} \tan \beta\left[\left(-2+\frac{3}{C^{1}}\right)-\alpha\left(1-\frac{1}{C^{1}}\right)\left[-2+\frac{c}{a_{c}}+\left(\frac{c}{a_{c}}\right)^{2}\right]\right]
$$

Replacing variable $a$ with $a_{c}$ in Eq. (13):

$$
\Delta V_{c}=2 \pi a_{c}^{3}(2-\tan \beta) \frac{(1-2 v)}{E} Y \ln \frac{c}{a_{c}}
$$

The volume loss in the plastic zone and elastic zone is calculated starting from $u_{r=a_{c}}$. To simplify the calculation, it is assumed that $u_{r=a_{c}}$ is calculated for a spherical cap defined by the $\psi$ angle shown in Fig. 2(a). It thus follows that:

$$
\Delta V_{e}+\Delta V_{p}=2 \pi a_{c}^{2}(1-\cos \Psi) u_{r=a_{c}}
$$

Replacing variable $\cos \psi$ with $\left(h_{c}-h\right) / c$ leads to: 


$$
\Delta V_{e}+\Delta V_{p}=2 \pi a_{c}^{2}\left(1-\frac{a_{c}}{c} \tan \beta\left(1-\frac{1}{C^{1}}\right)\right) \frac{Y}{E} a_{c}\left[(1-v)\left(\frac{c}{a_{c}}\right)^{3}-\frac{2}{3}(1-2 v)\left(1+3 \ln \frac{c}{a_{c}}\right)\right]
$$

Taking $\Lambda$ 'defined by Eq. (15) and substituting Eqs. (22), (23) and (25) into Eq. (19) leads to:

$$
\begin{aligned}
& \frac{1}{3} \Lambda^{\prime}\left[\left(-2+\frac{3}{C^{1}}\right)-\alpha\left(1-\frac{1}{C^{1}}\right)\left[-2+\frac{c}{a_{c}}+\left(\frac{c}{a_{c}}\right)^{2}\right]\right]= \\
& \left(\frac{c}{a_{c}}\right)^{3}-\frac{2}{3} \frac{(1-2 v)}{(1-v)}+\tan \beta\left[-\left(1-\frac{1}{C^{1}}\right) \cdot\left(\frac{c}{a_{c}}\right)^{3}+\frac{(1-2 v)}{(1-v)}\left[\frac{2}{3} \cdot\left(1-\frac{1}{C^{1}}\right)+\left(1-\frac{2}{C^{1}}\right) \ln \left(\frac{c}{a_{c}}\right)\right]\right]
\end{aligned}
$$

When $\tan \beta<<1$, Eq. (26) becomes:

$$
\left(\frac{c}{a_{c}}\right)^{3}=\frac{1}{3} \Lambda^{\prime}\left[\left(-2+\frac{3}{C^{1}}\right)-\alpha\left(1-\frac{1}{C^{1}}\right)\left[-2+\frac{c}{a_{c}}+\left(\frac{c}{a_{c}}\right)^{2}\right]\right]+\frac{2}{3} \frac{(1-2 v)}{(1-v)}
$$

This equation is more complex than the Johnson's formulation. The determination of the $c / a_{c}$ ratio necessitates the solution of a polynomial equation of degree 3 and the knowledge of the $C^{l}$ parameter. FEM simulations show that the results obtained from Eq. (27) are very close to those obtained from Eq. (26), even for large values of $\beta$. Notice that Eq. (27) becomes equal to the Johnson's formulation (Eq. (5)) if there is no pile-up or sink-in.

\section{B. Materials that exhibit sink-in}

The concept of ECM based on the equality between the displaced volumes and the volume loss in the indented material is applied for materials that exhibit sink-in.

Fig. 3 shows that the geometrical constructions proposed to define the pile-up can be used to define the sink-in. 
FIG. 3 : Volume displaced by the indented materials that exhibit sink-in.

\begin{abstract}
In light of this analogy, the volume of the sink-in, i.e. $\Delta V_{s i}$, can be determined with Eq. (19) where $\Delta V_{p u}$ is replaced by $\Delta V_{s i}$. It thus follows that the $c / a_{c}$ ratio is also obtained from Eq. (27) in the case of materials that exhibit sink-in.
\end{abstract}

\title{
5. APPLICATION OF THE NEW ECM
}

To illustrate the new ECM developed in the preceding section, the constraint factor $C_{f}=H / Y$ obtained by applying Eqs. (18) and (27) is compared with numerical results.

\section{A. FEM. model}

The finite element analysis presented here assumes a conical perfectly rigid indenter in frictionless contact with the flat surface of the specimen. The simulations were performed in axisymmetric mode using the large strain elastic-plastic feature of the Abaqus finite element code.

For conical indentation of elastic-ideally plastic materials, the degree of indentation is commonly defined by the non-dimensional $E^{*} \tan \beta / Y\left(\right.$ where $\left.E^{*}=\mathrm{E} /\left(1-v^{2}\right)\right) .{ }^{2}$ The numerical simulations were performed for various $\beta$ angles and constant $E / Y$ ratio. The $E / Y$ ratios of the indented materials are equal to 200, 2000, 20,000 and 200,000 . The high value of $E / Y$ ratio $(200,000)$ was chosen in order to obtain a material very similar to the rigid-ideally plastic material for which the similarity regime can be reached. The finite element simulations were performed for materials with Poisson's values $v=0,0.3$ and 0.5 . The constitutive model of the elastic-ideally plastic indented material was taken to follow the well known $J_{2^{-}}$ associated flow theory with rate-independent deformation. The constraint factor is calculated starting from 
the weight average of the indentation pressures obtained during the indentation process. The size of the elements in contact with the indenter is equal to $0.003 a$ at maximum penetration depth. Details of the finite element meshes and their density-region hierarchy are given by Bartier and Hernot. ${ }^{17}$

\section{B. FEM results}

Fig. 4 shows the development of $C_{f}$ and $C^{l}$ with $\Lambda^{\prime}$. Four regimes are differentiated in this figure. First, up to $\Lambda^{\prime}=0.91$, there is no deviation in behaviour from the elastic stage described by Love ${ }^{1}$. This first regime can be called "pseudo-elastic regime". The second regime occurs when the contact pressure lies between approximately $0.7 Y$ and $3 Y$. This regime corresponds to the elastic-plastic regime defined by Johnson in which the Johnson's ECM given in Eq. (18) is considered as valid., ${ }^{2,8,10}$ The third regime is reached when $C_{f}=3$. This regime is commonly called "fully plastic". Notice that $C^{l}$ is not constant in this regime. We will show thereafter that this regime is not fully plastic because the elasticity can not be considered as negligible. The fourth regime is reached for large values of $\Lambda^{\prime}$ and corresponds to a drop of $C_{f}$. We will show that this regime is fully plastic.
(a): $C_{f^{-}} \Lambda^{\prime}$ curves
(b): $C^{l}-\Lambda^{\prime}$ curves

FIG. 4 : Evolution of the constraint factor, $C_{f}$, and $C^{l}$ parameter during conical indentation of elastic-ideally plastic material of large $E / \sigma_{y}$ ratio. 


\section{New ECM predictions}

To calculate the radius of the plastic zone from Eq. (27), one has to define the value of the $\alpha$ constant. If the existence of a similarity regime is assumed, it is also assumed that $C_{f}$ and $C^{l}$ take constant value during this regime. For materials very similar to the rigid-ideally plastic material, $C_{f}$ and $C^{l}$ remain constant for large values of $\Lambda^{\prime}$. In order not to obtain $c \rightarrow \infty$ from Eq. (27), it is necessary to verify that:

$$
\alpha=\frac{3-2 C^{1}}{C^{1}-1} \frac{1}{-2+\frac{c}{a_{c}}+\left(\frac{c}{a_{c}}\right)^{2}}
$$

For Alcala et al. ${ }^{18}, C^{1}=1.202$, for Felder et al. ${ }^{19}, C^{1}=1.263$, for Malherbe et al. ${ }^{20}, C^{1}=1.28$ and for Pelletier $^{21}, C^{1}=1.3$. For the proposed study, $C^{1}$ was fixed at 1.28 in Eq. (27) (value close to the numerical results).

In the Johnson's $\mathrm{ECM}^{8}$, Eq. (18) gives the elastic-plastic boundary at $c / a_{c} \approx 2.3$ when the "fully plastic" state, defined by $C_{f}=3$, occurs. This result matches the experimentally based estimates of Zielinski et al. ${ }^{22}$ who found that $c / a_{c} \approx 2.12$. By assuming that $c / a_{c} \approx 2.2$ and $C^{1}=1.285$, Eq. (28) gives $\alpha=0.3$. This value is in agreement with the numerical results obtained for the studied materials. For example, the calculation of the pile-up volume for a material with $E / Y=2,000$ and $\tan \beta=0.1$ gives $\alpha=0.3082$ (Fig. 5)

FIG. 5: (a): plastic zone, (b): shape of the imprint obtained from FEM (material with $E / Y=2,000$ and $\tan \beta=0.1$ ).

$\alpha=0.3$ was introduced in Eq. (27).

The variation of the normalized indentation pressure $(H / Y)$ with the degree of indentation $\left(\Lambda^{\prime}\right)$ obtained from Eqs. (18) and (27) is illustrated in Fig. 5. 
FIG. 6: Predictions from the new ECM for conical indentation of elastic-ideally materials with $E / Y=200,000$.

In this figure, $H / Y$ was calculated for the material with $E / Y=200,000$. This figure reveals that the theoretical results are in accordance to the finite element simulations in the third indentation regime and in the final portion of the second indentation regime. Fig. 7(a) shows that the shape of the plastic zone is very different from the shape described in Fig. 2 at the beginning of the elastic-plastic regime. For $\Lambda^{\prime}=2$, the plastic zone remains embedded within an elastic zone. The failure of the assumptions involved in ECM can explain the difference between theoretical results and numerical results at the beginning of the elasticplastic regime. Fig. 6(b) shows that the plastic zone is similar to the shape of the plastic zone described in Fig. 2 when $\Lambda^{\prime}=10$. Starting from this value, the predicted constraint factor curve fits well with all numerical data points up to the end of the third indentation regime.
(a): $\Lambda^{\prime}=2$
(b): $\Lambda^{\prime}=10$

FIG. 7: Plastic zone in a material, with $E / Y=200,000$ and $v=0$, indented by cone; (a): $\Lambda^{\prime}=2,(\mathrm{~b}): \Lambda^{\prime}=10$.

Fig. 4(a) shows that the constraint factor remains about constant and close to 3 for $\Lambda^{\prime}$ in the range 362000 (third regime). It is commonly assumed that "fully plastic" regime is reached when the constraint factor remains constant during plastic deformation. It is also assumed that "fully plastic" regime occurs when the elastic contribution to the strain field beneath the indenter is negligible, and the parameters $\mathrm{E}^{*} \tan \beta / \mathrm{Y}$ or $\Lambda^{\prime}$ cease to uniquely define the degree of indentation. In order to determine if the elastic contribution to the strain field beneath the indenter is negligible when $C_{f}=3$, simulations were performed for materials with values of $v=0,0.3$ and 0.5 . 
Fig. 4(b) shows that the development of the pile-up is dependent on the Poisson's ratio when $C_{f}=3$. This result shows that $\Lambda^{\prime}$ continues to define the degree of indentation, when $C_{f}$ remains constant. Thus, the third regime can not be considered as "fully plastic". For this regime, the new ECM has the capacity to describe the indentation test (Fig. 6).

As it was mentioned above, the fourth regime corresponds to a drop of $C_{f}$. The normalized indentation pressure $(H / Y)$ obtained from the proposed ECM also decreases in the fourth indentation regime, but this decrease is much less pronounced (Fig. 5). Fig. 4(b) shows that the decrease in $C_{f}$ corresponds to a steeper increase in the pile-up.

When $C_{f}$ begins to decrease from 3 , the $C_{f} \Lambda^{\prime}$ and $C^{l}-\Lambda^{\prime}$ curves markedly separate and thus $\Lambda^{\prime}$ is inappropriate to correlate the indention tests on materials of various Poisson's ratios. Fig. 8 shows the existence of unique solution if $C_{f}$ and $C^{l}$ are plotted versus $\tan \beta$. This indicates that the elastic contribution to the strain field beneath the indenter is negligible for the regime where $C_{f}$ decreases. Thus, this regime can be considered as "fully plastic". For this regime, the new ECM is inappropriate to describe the indentation test.
(a): $C_{f^{-}} \tan \beta$ curves
(b): $C^{l}-\tan \beta$ curves

FIG. 8: Variation of $C_{f}$, and $C^{l}$ with $\tan \beta$ for an elastic-ideally plastic material of large $E / Y$ ratio.

Fig. 9 shows the Variation of $C_{f}$, with $\Lambda^{\prime}$ for elastic-ideally plastic materials of various $E / Y$ ratios. This figure shows that with decreasing $E / Y$ ratio the regime where hardness remains constant tends to vanish. The same phenomenon was observed in the case of spherical indentation of elastic-ideally plastic materials. $^{17}$

FIG. 9: Predictions from the new ECM for conical indentation of various elastic-ideally materials. 
As shown above, the regime where $C_{f}$ decays is fully plastic. The existence of the fully plastic regime is due to the high values of the plastic strain located in periphery of the contact and to the greatest facility of material displacement on the surface. The combination of these two phenomena has as a consequence a decrease in the mean contact pressure and a higher increase in the pile-up (Fig. 4). Fig. 9 also shows that for a given apical angle of the tip, $C_{f}$ becomes constant irrespectively of $\Lambda^{\prime}$ if fully plastic regime is reached. For our conditions, $C_{f} \approx 2.54$ if a Vickers indenter with an equivalent conical tip half apex angle of $70: 3^{\circ}$ is used. Notice that $C_{f}$ will be higher than this value in fully plastic regime for friction contact and strain hardening materials. ${ }^{23,24}$ As for the elastic-ideally plastic material of large $E / Y$ ratio, ECM does not match numerical results in the regime where $C_{f}$ decays (Fig. 9). We can notice however that the new ECM gives better results than the Johnson's ECM because pile-up or sink-in is taken into account.

\section{CONCLUSION}

A new expanding cavity model (ECM) for describing conical indentation of elastic ideally-plastic material was proposed in this paper. We show that the Johnson's expanding cavity model based on the conservation of volume during an increment of penetration can be replaced by an ECM based on the equality between the displaced volumes and the volume loss in the indented material. For the proposed ECM, it is assumed that the volume of material displaced by the indenter is equal to the volume loss, due to elastic deformation, in the material and depends on the pile-up or sink-in. It was shown that the proposed ECM matches very well numerical data in the final portion of the transition regime for which the contact pressure lies between approximately $2.5 \mathrm{Y}$ and $3 Y$. For material of large $E / Y$ ratio, the new ECM also matches very well numerical data in the plastic-similarity regime (regime in which $C_{f}=3$ ). It is commonly assumed that this last regime is "fully plastic". We show that it is not the case because the 
elastic contribution to the strain field beneath the indenter is non-negligible in this regime. For material of smaller $E / Y$ ratio, the proposed ECM gives better results than the Johnson's ECM because pile-up or sinkin is taken into account.

\section{REFERENCES}

1. A-E-H. Love : Boussinesq's problem for a rigid cone. Qart. J. Math. 10, 161 (1939).

2. K-L. Johnson: Contact mechanics (Cambridge University Press, Cambridge, England, 1985), pp. 171.

3. D. Tabor: The Hardness of Metals (Clarendon Press, Oxford, England, 1951), pp. 101.

4. M. Mata and J. Alcala: Mechanical property evaluation through sharp indentations in elastoplastic and fully plastic contact regimes. J. Mater. Res., 18, 1705 (2003).

5. D-M. Marsh: Plastic flow in glass. Proc. Roy. Soc. A, 279, 420 (1964).

6. X-L. Gao : New expanding cavity model for indentation hardness including strain-hardening and indentation size effects. J. Mater., 21, 1317 (2006).

7. M. Mata, O. Casals and J. Alcala: The plastic zone size in indentation experiments: The analogy with the expansion of a spherical cavity. Int. J. Solids Struct. 43, 5994 (2006).

8. K-L. Johnson: The correlation of indentation experiments. J. Mech. Phys. Solids 18, 115 (1970).

9. R. Hill: The mathematical theory of plasticity (Oxford University Press, London, England, 1950).

10. C-J. Studman, M-A. Moore and S-E. Jones: On the correlation of indentation experiments. J. Phys. D appl. Phys. 10, 949 (1977).

11. S-S. Chiang, D-B. Marshall and A-G. Evans. The response of solids to elastic/ plastic indentation. I. Stresses and residual stresses. J. Appl. Phys. 53, 298 (1982).

12. G. Feng, S. Qu, Y. Huang and W-D. Nix: An analytical expression for the stress field around an elastoplastic indentation/contact. Acta Mater. 55, 2929 (2007).

13. E-H. Yoffe: Elastic stress fields caused by indenting brittle materials. Phil. Mag. A. 46, 617 (1982).

14. A-C. Fischer-Cripps: Elastic-plastic behaviour in materials loaded with a spherical indenter. J. Mat. Sci. 32, 727 (1997). 
15. D. Kramer, H. Huang, M. Kriese, J. Robach, J. Nelson, A. Wright, D. Bahr and W-W. Gerberich: Yield strength predictions from the plastic zone around nanocontacts. Acta mater. 47, 333 (1999).

16. X. Hernot, F. Pichot: Influence du coefficient de Poisson sur les régimes d'indentation sphérique. Mat. Tech. 96, 31 (2009).

17. O. Bartier and X. Hernot: Etude des régimes de déformation de matériaux élastiques parfaitement plastiques au cours de l'indentation parabolique et sphérique. Mat. Tech. 96, 20 (2009).

18. J. Alcala, A.C. Barone and M. Anglada: The influence of plastic hardening on surface deformation modes around Vickers and spherical indents. Acta mater. 48, 3451 (2000).

19. E. Felder: Analytical correlation of indentation experiments. Phil. Mag. 86, 5239 (2006).

20. S. Malherbe, S. Benayoun, S. Morel and A.Iost: Caractérisation mécanique de matériaux élastoplastiques - utilisation d'indenteurs axisymétriques. Mat. Tech. 93, 213 (2005).

21. H. Pelletier : Étude de la formation du bourrelet autour des empreintes de nanoindentation. Mat. Tech. 93, 229 (2005).

22. W. Zielinski, H. Huang, and W-W. Gerberich: Microscopy and microindentation mechanics of single crystal Fe-3 wt. \% Si: Part II TEM of the indentation plastic zone. J. Mater. Res. 8, 1300 (1993).

23. M. Mata and J. Alcala: The role of friction on sharp indentation. J. Mech. Phys. Solids. 52, 145 (2004).

24. X-L. Gao: An expanding cavity model incorporating strain-hardening and indentation size effects. Int. J. Solids Struct. 43, 6615 (2006). 
FIG. 1: Schematic illustrating the Johnson's ECM for conical indentation.

FIG. 2: Schematic illustrating elastic-plastic indentation as idealized by the expanding cavity model considering surface deformation. (a) pile-up and (b) sink-in.

FIG. 3: Volume displaced by the indented materials that exhibit sink-in.

FIG. 4: Evolution of the constraint factor, $C_{f}$, and $C^{l}$ parameter during conical indentation of elasticideally plastic material with $E / Y=200,000$. (a): $C_{f}$, (b): $C^{l}$.

FIG. 5: (a): plastic zone, (b): shape of the imprint obtained from FEM (material with $E / Y=2,000$ and $\tan \beta=0.1$.

FIG. 6: Predictions from the new ECM for conical indentation of elastic-ideally materials with $E / Y=200,000$.

FIG. 7: Plastic zone in a material, with $E / Y=200,000$ and $v=0$, indented by cone; (a): $\Lambda^{\prime}=2,(\mathrm{~b}): \Lambda^{\prime}=10$. FIG. 8: Variation of $C_{f}$, and $C^{l}$ with $\tan \beta$ for an elastic-ideally plastic material with $E / Y=200,000$. (a): $C_{f}$, (b): $C^{l}$.

FIG. 9: Predictions from the new ECM for conical indentation of various elastic-ideally materials. 


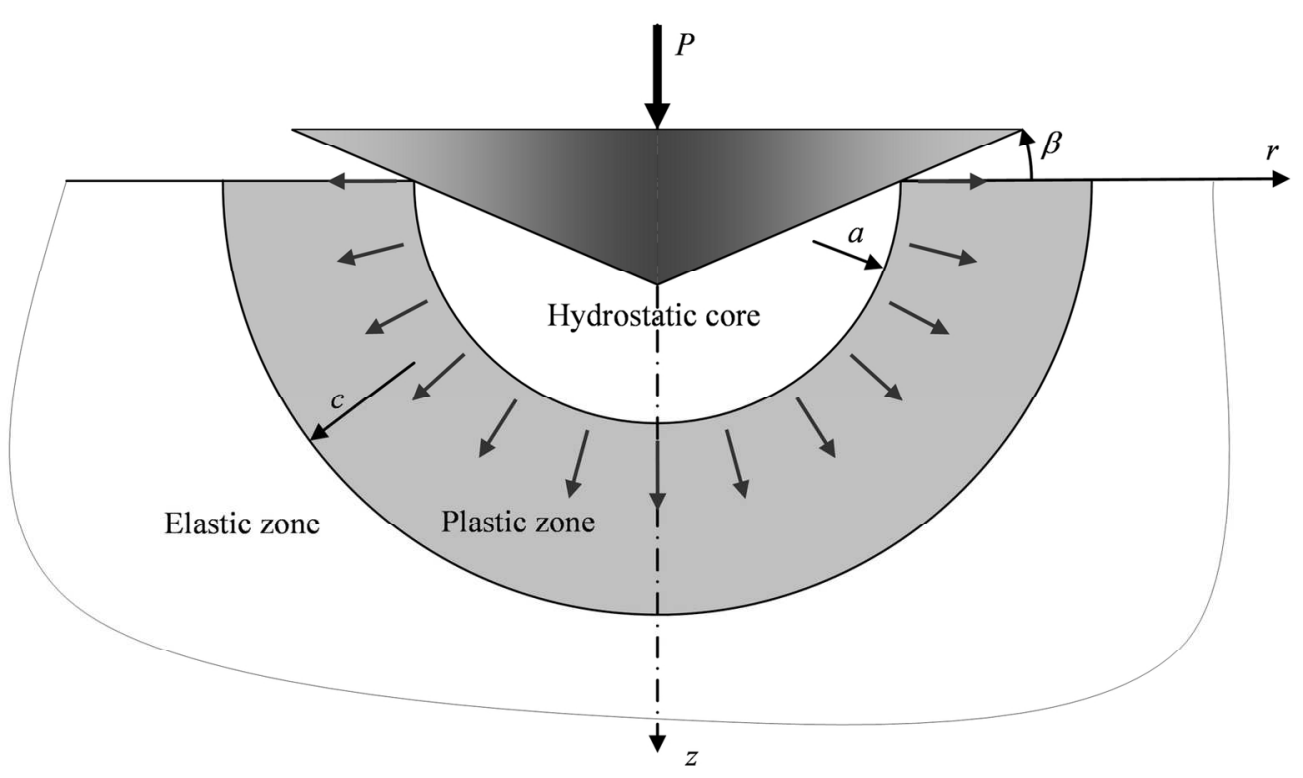

FIG. 1: Schematic illustrating the Johnson's ECM for conical indentation. $72 \times 42 \mathrm{~mm}(600 \times 600 \mathrm{DPI})$ 


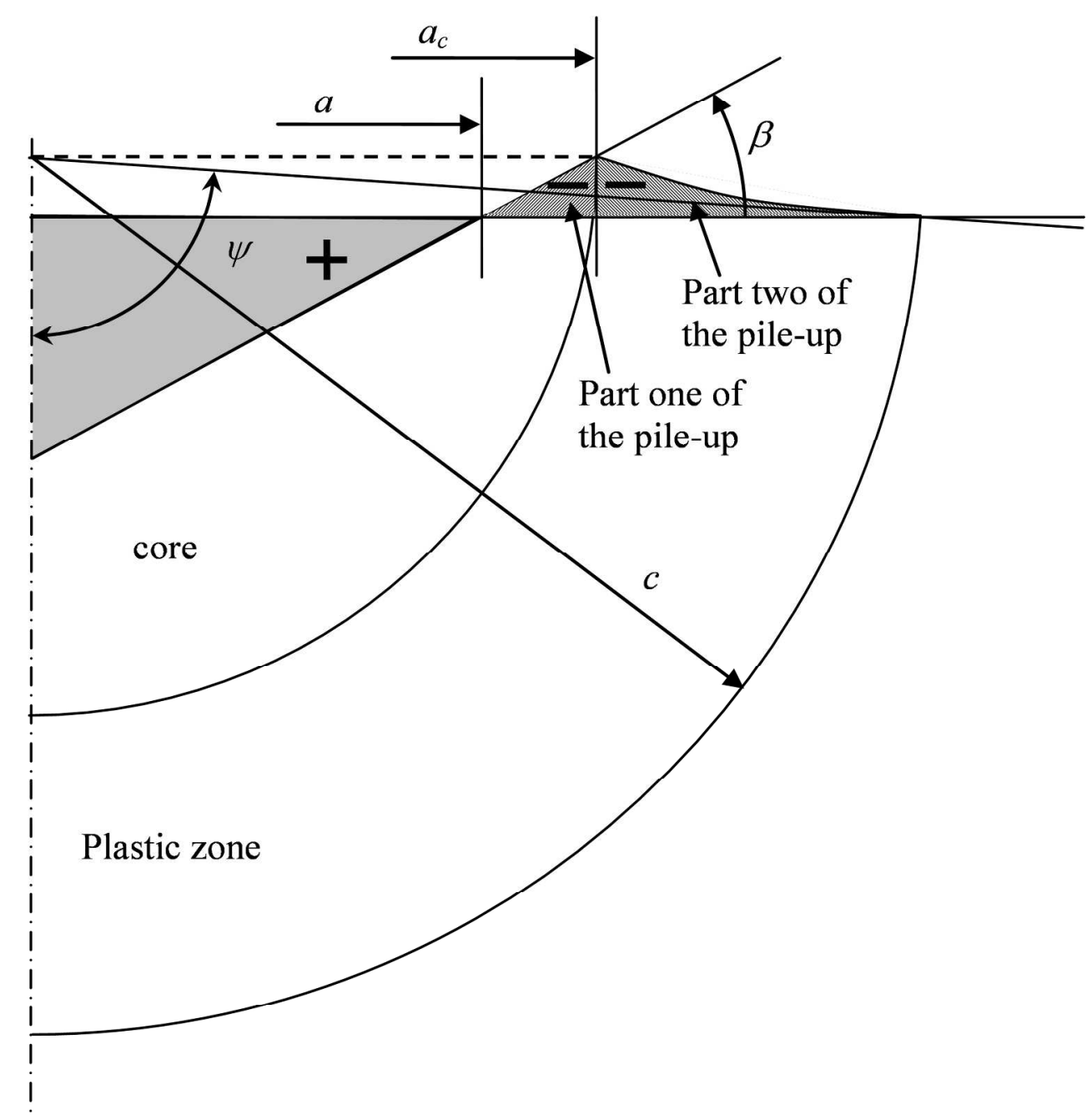

FIG. 2: Schematic illustrating elastic-plastic indentation as idealized by the expanding cavity model considering pile-up. (a) pile-up and (b) sink-in. $128 \times 133 \mathrm{~mm}(600 \times 600 \mathrm{DPI})$ 
FIG. 2: Schematic illustrating elastic-plastic indentation as idealized by the expanding cavity model considering pile-up. (a) pile-up and (b) sink-in. $108 \times 93 \mathrm{~mm}(600 \times 600 \mathrm{DPI})$ 

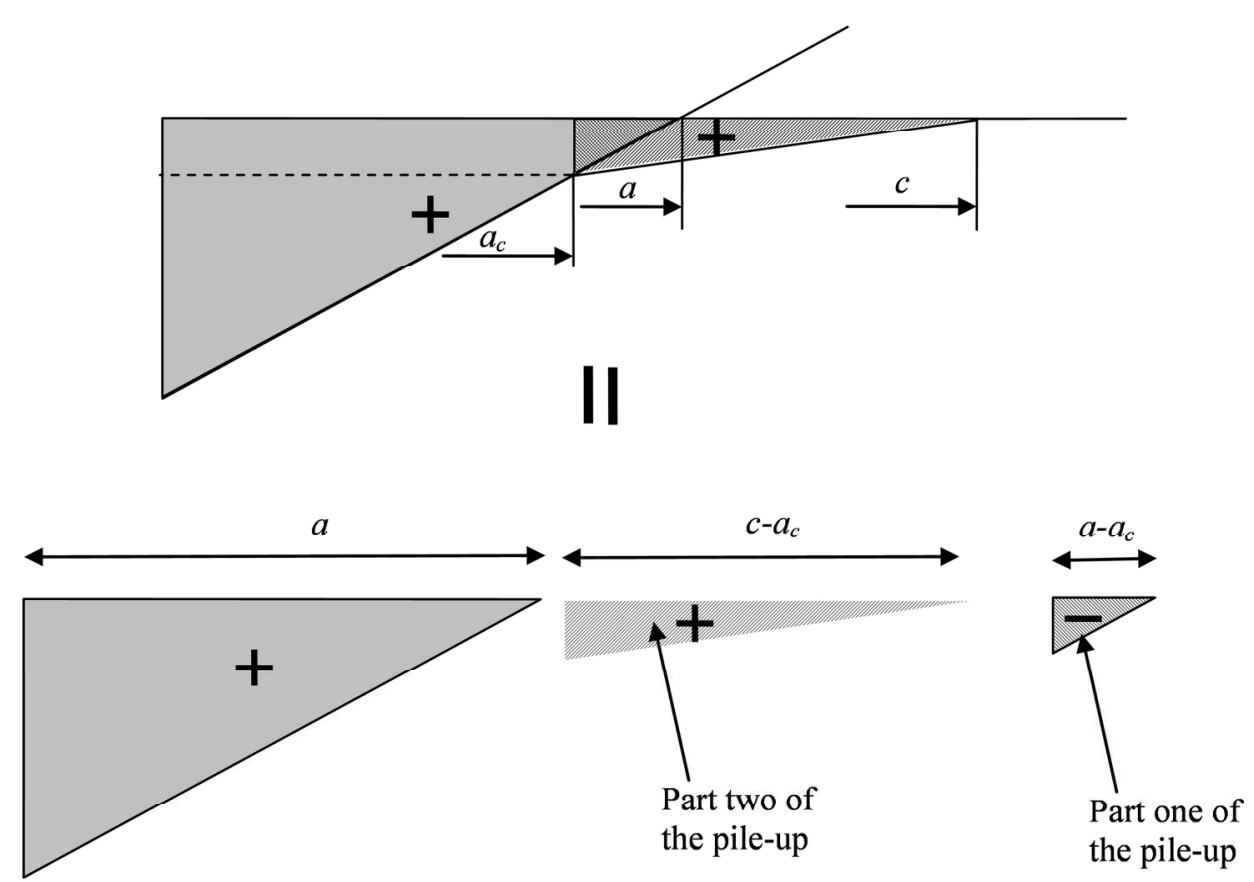

FIG. 3 : Volume displaced by the indenter materials that exhibit sink-in. $89 \times 64 \mathrm{~mm}(600 \times 600$ DPI $)$ 


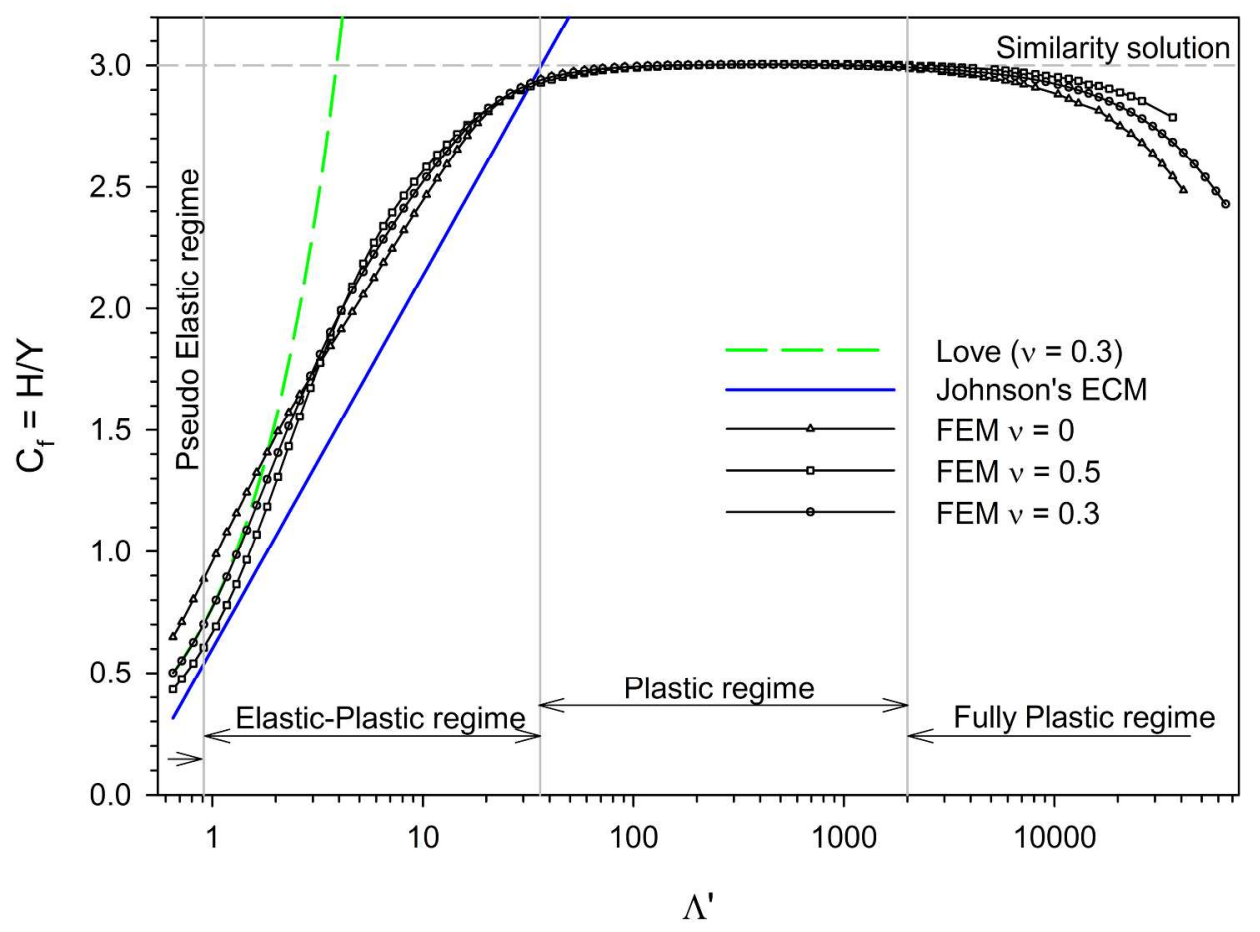

FIG. 4 : Evolution of the constraint factor, $C_{f}$, and $C^{1}$ parameter during conical indentation of elastic-ideally plastic material of large $E / Y$ ratio. $119 \times 95 \mathrm{~mm}(600 \times 600 \mathrm{DPI})$ 


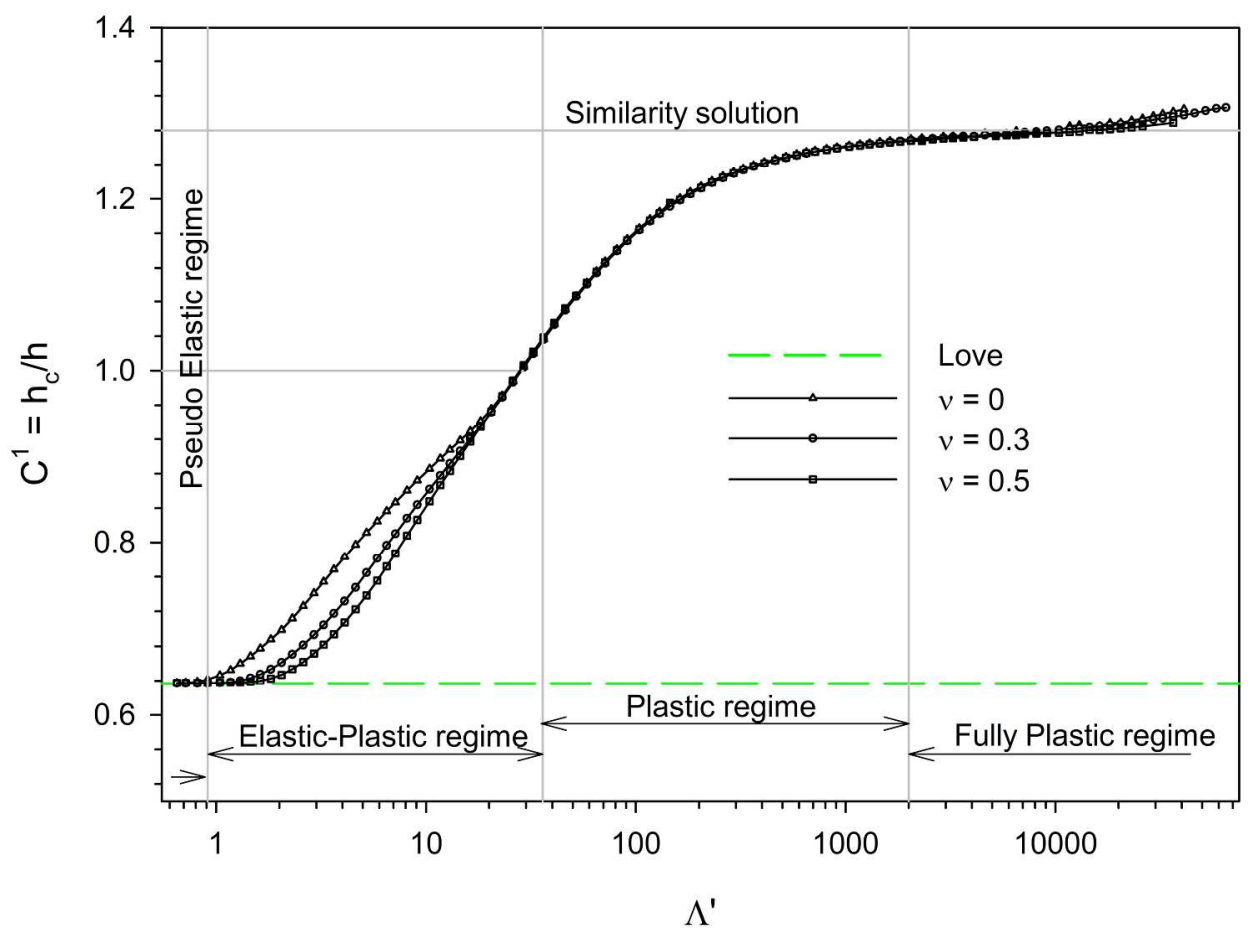

FIG. 4 : Evolution of the constraint factor, $C_{f}$, and $C^{1}$ parameter during conical indentation of elastic-ideally plastic material of large $E / Y$ ratio. $119 \times 95 \mathrm{~mm}(600 \times 600 \mathrm{DPI})$ 
FIG. 5: (a): plastic zone, (b): shape of the imprint obtained from FEM (material with $E / Y=2,000$ and $\tan \beta=0.1$. $107 \times 83 \mathrm{~mm}(600 \times 600 \mathrm{DPI})$ 


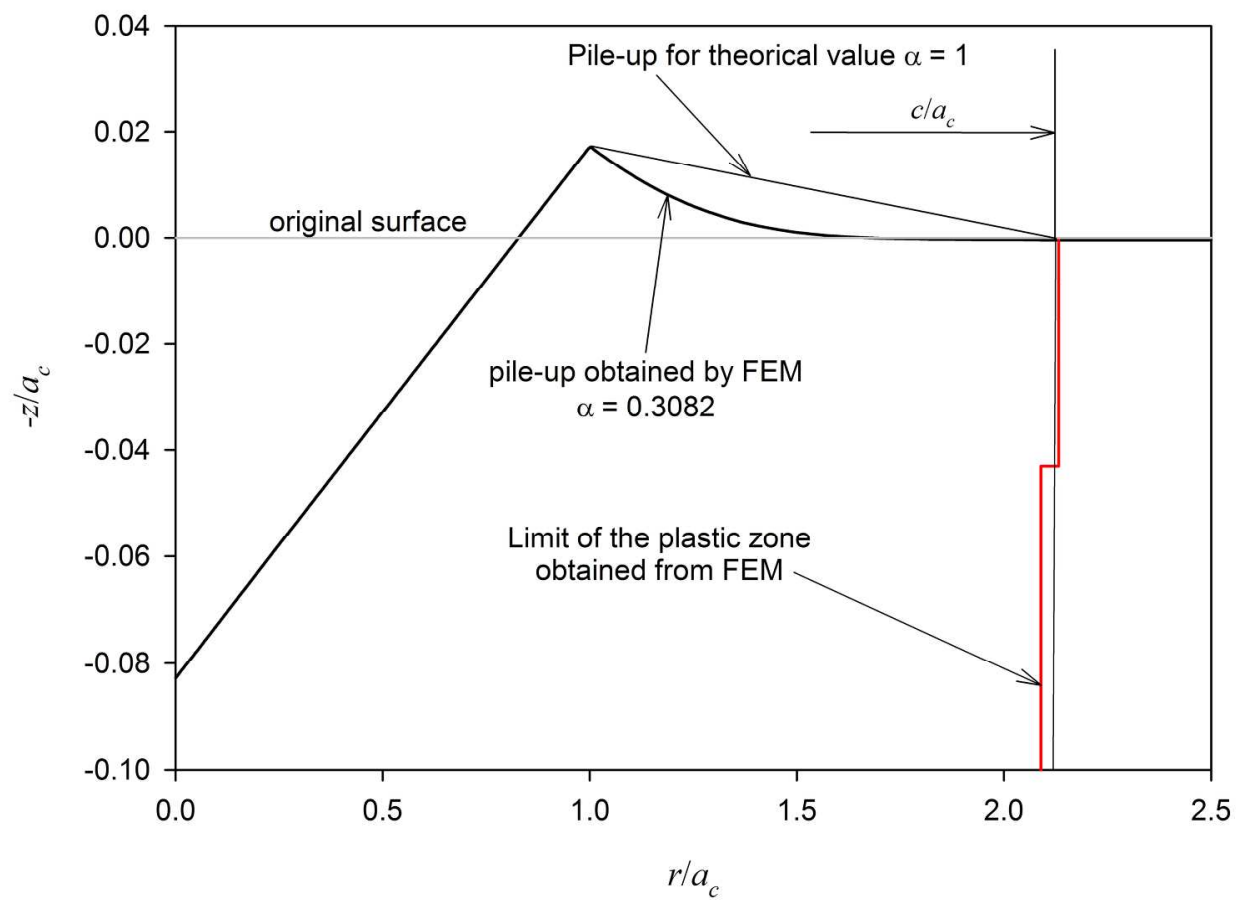

FIG. 5: (a): plastic zone, (b): shape of the imprint obtained from FEM (material with $E / Y=2,000$ and $\tan \beta=0.1$ ). $106 \times 81 \mathrm{~mm}(600 \times 600 \mathrm{DPI})$ 
1

2

3

4

5

6

7

8

9

10

11

12

13

14

15

16

17

18

19

20

21

22

23

24

25

26

27

28

29

30

31

32

33

34

35

36

37

38

39

40

41

42

43

44

45

46

47

48

49

50

51

52

53

54

55

56

57

58

59

60

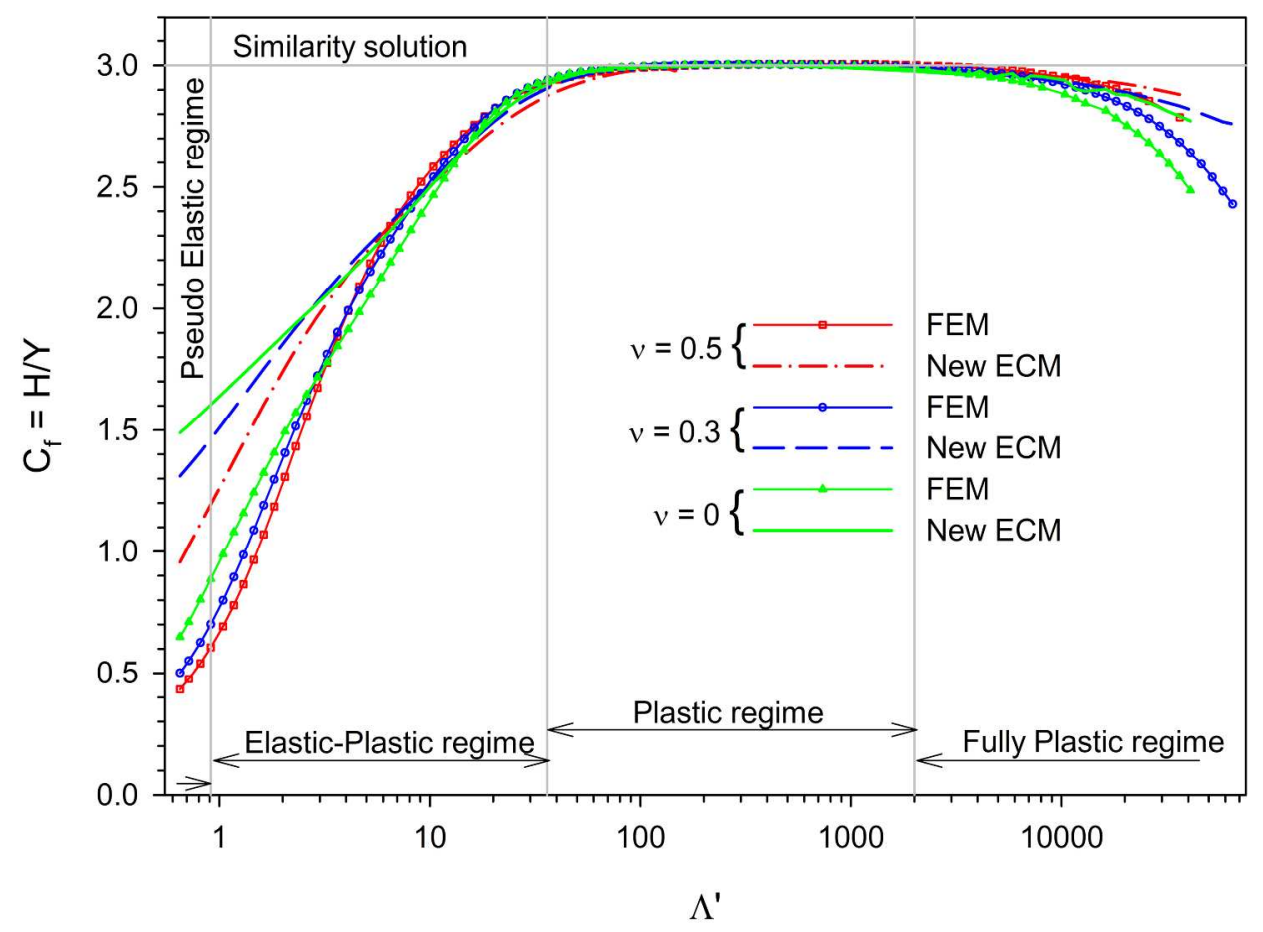

FIG. 6: Predictions from the new ECM for conical indentation of elastic-ideally materials with $E / Y=200,000$. $119 \times 95 \mathrm{~mm}(600 \times 600 \mathrm{DPI})$ 
FIG. 7: Plastic zone in a material, with $E / Y=200,000$ and $v=0$, indented by cone; (a): $\Lambda^{\prime}=2,(b): \Lambda^{\prime}=10$. $107 \times 90 \mathrm{~mm}(600 \times 600$ DPI $)$ 
FIG. 7: Plastic zone in a material, with $E / Y=200,000$ and $v=0$, indented by cone; $(a): \Lambda^{\prime}=2,(b): \Lambda^{\prime}=10$. $107 \times 91 \mathrm{~mm}(600 \times 600 \mathrm{DPI})$

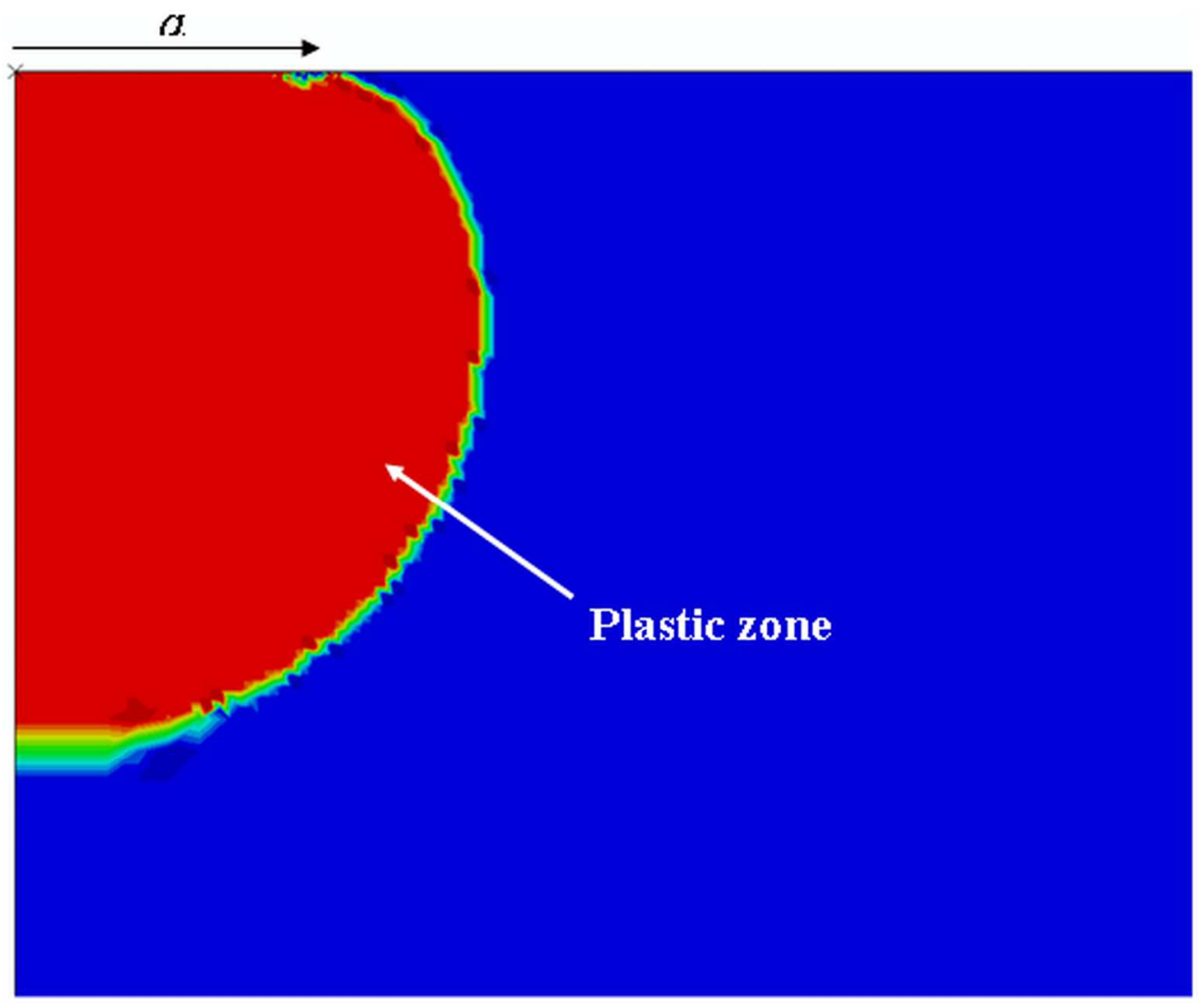




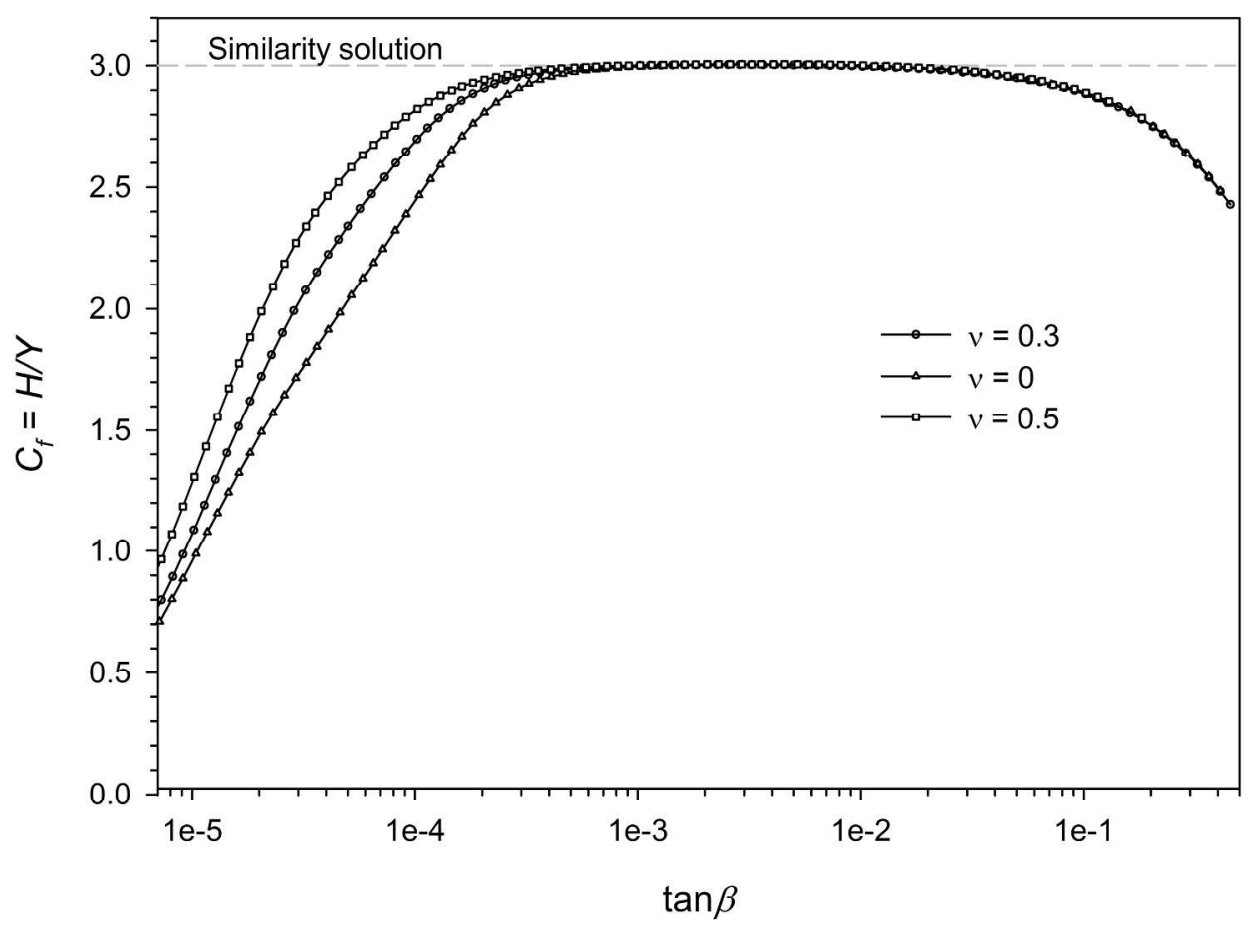

FIG. 8: Variation of $C_{f}$, and $C^{1}$ with $\tan \beta$ for an elastic-ideally plastic material with $E / Y=200,000$. (a): $C_{f},(b)$ : $C^{1}$. $119 \times 94 \mathrm{~mm}(600 \times 600$ DPI $)$ 
1

2

3

4

5

6

7

8

10

11

12

14

15

16

17

18

19

20

21

22

23

24

25

26

27

28

29

30

31

32

33

34

35

36

37

38

39

40

41

42

43

44

45

46

47

48

49

50

51

52

53

54

55

56

57

58

59

60

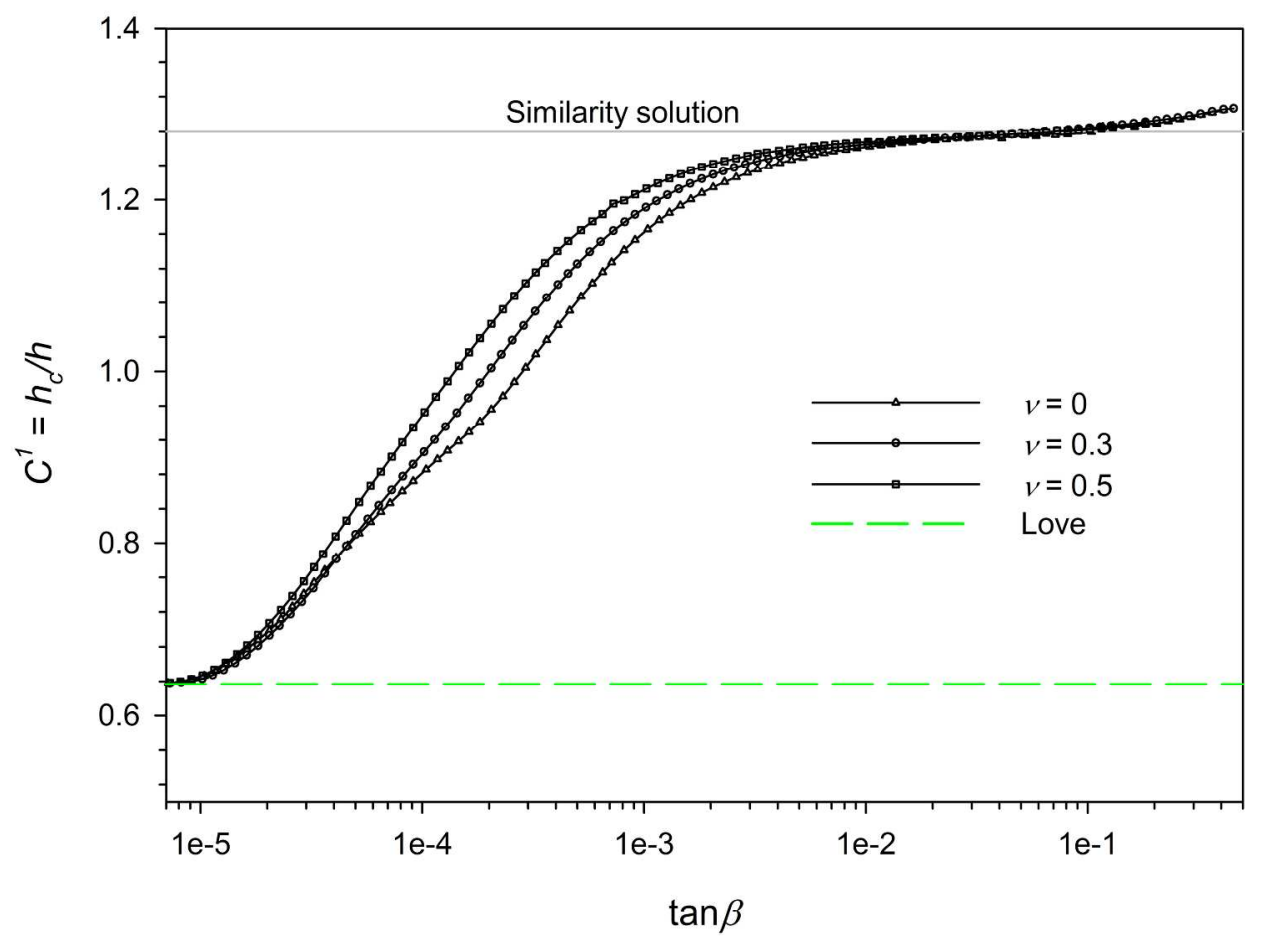

FIG. 8: Variation of $C_{f}$, and $C^{1}$ with $\tan \beta$ for an elastic-ideally plastic material with $E / Y=200,000$. (a): $C_{f}$, (b): $\mathrm{C}^{1}$. $119 \times 95 \mathrm{~mm}(600 \times 600 \mathrm{DPI})$ 


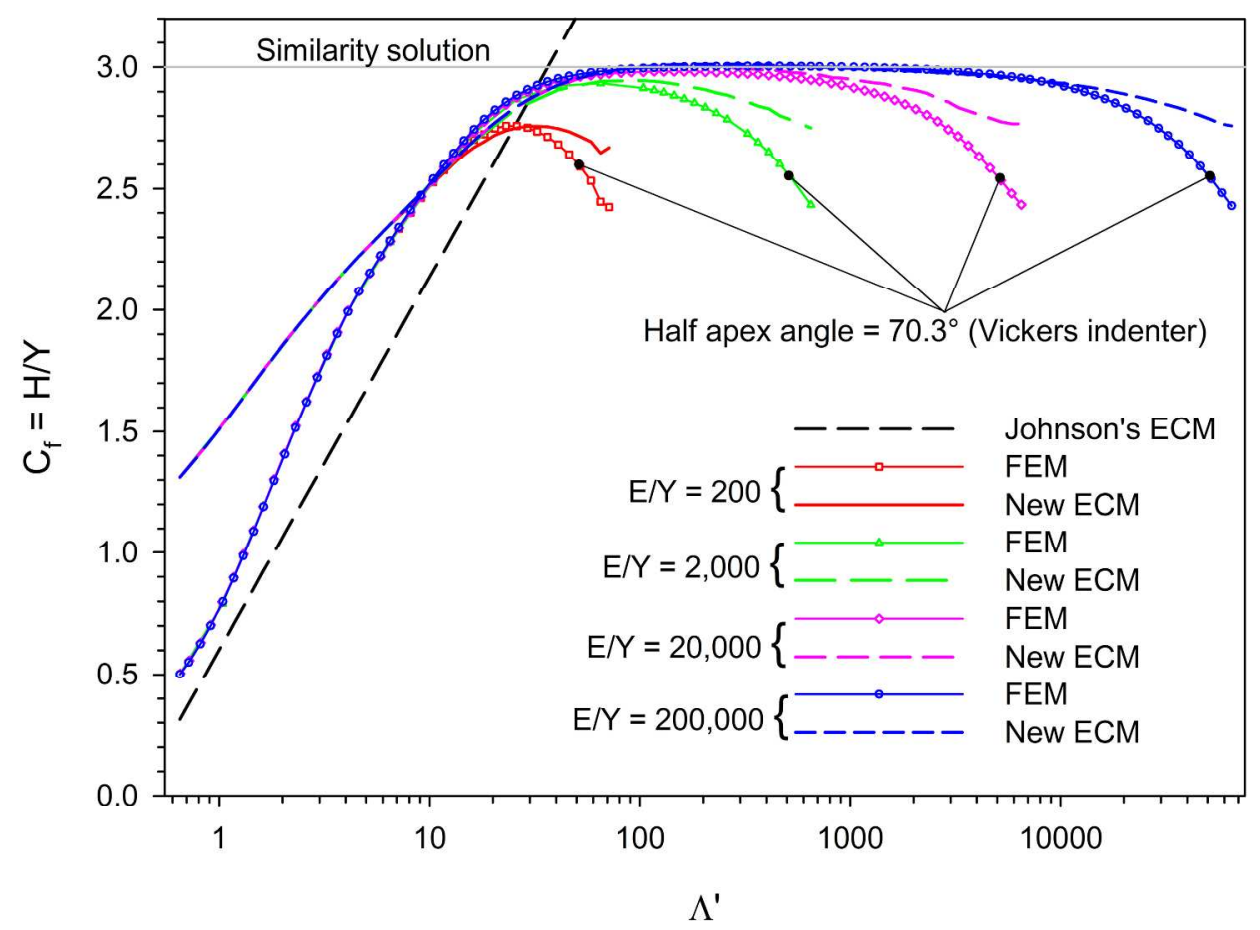

FIG. 9: Predictions from the new ECM for conical indentation of various elastic-ideally materials. $119 \times 95 \mathrm{~mm}(600 \times 600 \mathrm{DPI})$ 